\title{
ON A RELATION BETWEEN THE TOPOLOGY AND THE INTRINSIC AND EXTRINSIC GEOMETRIES OF A COMPACT SUBMANIFOLD
}

\author{
by PUI-FAI LEUNG
}

(Received 5th June 1984)

\section{Introduction}

Let $M^{n}$ be an $n$-dimensional smooth compact Riemannian manifold. By a theorem of Nash, we can think of it as an isometrically immersed submanifold in some higher dimensional Euclidean space $\mathbb{R}^{n+m}$. Viewing in this way we can compare the intrinsic geometry of $M$ to its extrinsic geometry. Classically, the Gauss equation

$$
K(X, Y)=\langle B(X, X), B(Y, Y)\rangle-\|B(X, Y)\|^{2}
$$

where $K(X, Y)$ denotes the sectional curvature in $M$ corresponding to the plane spanned by the two orthonormal vectors $X, Y$ and $B$ denotes the second fundamental form gives one of the most important relations between the intrinsic and extrinsic geometries of $M$. In this note we shall prove the following.

Theorem. Let $M^{n}$ be a smooth, compact, isometrically immersed submanifold in a Euclidean space $\mathbb{R}^{n+m}$. If for all $p$ in $M$ and all orthonormal vectors $X, Y$ in $T M_{p}$, we have

$$
K(X, Y)>\|B(X, Y)\|^{2}
$$

then $M^{n}$ is homeomorphic to a sphere.

Suppose now we take an orthonormal basis $v_{1}, \ldots, v_{m}$ for the normal space at $p$ and let $A_{i}$ denote the shape operator corresponding to $v_{i}$, then we have

$$
\begin{aligned}
\|B(X, Y)\|^{2} & =\Sigma\left\langle B(X, Y), v_{i}\right\rangle^{2} \\
& =\Sigma\left\langle A_{i}(X), Y\right\rangle^{2}
\end{aligned}
$$

and so if $\|B(X, Y)\|^{2}$ is large, we would expect to have more terms in the summation and this means a larger codimension. Therefore it seems natural to expect that the codimension will be large when we have an immersion of a compact manifold with positive sectional curvature into an Euclidean space if the manifold is not homeomorphic to a sphere. This can be seen for example in the case of the real projective space with constant sectional curvature 1 immersed in an Euclidean space. 
Conversely, if we have a compact manifold with positive sectional curvature immersed in an Euclidean space with low codimension, then we would expect to find the topology of the manifold to be quite close to that of a sphere. This is indeed true and was proved by J. D. Moore [3]. More precisely, Moore proved that any compact manifold with positive sectional curvature immersed in an Euclidean space with codimension 2 must be a sphere topologically.

We shall prove our theorem by using the method of stable currents due to Lawson and Simons [2]. We shall show that under the hypothesis in our theorem, the manifold $M^{n}$ does not admit any stable currents of dimension $p$ with $1 \leqq p \leqq n-1$ and hence it follows that

$$
\pi_{1}(M)=0 \quad \text { and } \quad H_{1}(M, Z)=\cdots=H_{n-1}(M, Z)=0
$$

where $\pi_{1}(M)$ denotes the fundamental group and $H_{i}(M, Z)$ the $i$ th homology group with integer coefficients. From this it follows from the Hurewicz theorem and the Whitehead theorem that $M^{n}$ is a homotopy sphere ([4] p. 406, Theorem 25). Hence for the case $n \neq 3$, we conclude that $M$ is homeomorphic to a sphere. ( $n=2$ is well-known, $n=4$ due to Freedman, $n \geqq 5$ due to Smale.) For the case $n=3$, by a recent theorem due to Hamilton [1], we conclude that $M$ is a three dimensional spherical space form and hence since $\pi_{1}(M)=0$, it is homeomorphic to a sphere.

We remark that the non-existence of stable currents is a stronger property than the vanishing of homology group. For example on the surface in the shape of a dumb-bell there are stable closed geodesics and yet it is simply-connected. It seems natural to conjecture the following stronger form of the theorem of J. D. Moore.

Conjecture. There are no stable currents in a compact codimension two submanifold in the Euclidean space if the submanifold has positive sectional curvatures.

In the last section of this note, we shall discuss the above conjecture for the case of a hypersurface.

\section{Preliminaries [2]}

Let $M^{n}$ be a $n$-dimensional compact Riemannian manifold.

Denote by $\nabla$ the connection on $M$.

Let $\mathscr{S}$ be a $p$-dimensional current on $M$. We can define a Borel measure $\|\mathscr{S}\|$ on subsets of $M$ so that $\int_{M} d\|\mathscr{S}\|$ gives the "volume" of $\mathscr{S}$. In the following, all integrations will be over $M$ and with respect to this measure.

At almost every point $x \in \mathscr{S}$ we have a $p$-dimensional tangent space to $\mathscr{S}$ and we let $\left\{e_{1}, \ldots, e_{p}\right\}$ be an orthonormal basis. We extend this to $\left\{e_{1}, \ldots, e_{p}, n_{1}, \ldots, n_{q}\right\}$ an orthonormal basis of $T M_{x}$ so that $p+q=n$ and $n_{1}, \ldots, n_{p}$ are all normal to $\mathscr{S}$ at $x$.

We shall agree on the following range of indices and summation over repeated indice is assumed.

$$
\begin{aligned}
& 1 \leqq i, \quad j \leqq p \\
& 1 \leqq k, \quad l \leqq q
\end{aligned}
$$


Proposition 1. [2] If $\mathscr{S}$ is a stable p-dimensional current, then for any vector field $V$ which is the gradient of a function on $M$, we have

$$
\int\left\{\left(\Sigma\left\langle\nabla_{e_{i}} V, e_{i}\right\rangle\right)^{2}+2 \Sigma\left\langle\nabla_{e_{i}} V, n_{k}\right\rangle^{2}+\Sigma\left\langle R\left(V, e_{i}\right) V, e_{i}\right\rangle-\Sigma\left\|\nabla_{e_{i}} V\right\|^{2}\right\} \geqq 0
$$

where $R(V, X) V=\nabla_{V} \nabla_{X} V-\nabla_{X} \nabla_{V} V-\nabla_{[V, X]} V$ is the curvature tensor on $M$.

Proposition 2. [2] If there are no stable p-dimensional current in $M$, then $H_{p}(M, Z)=0$. In particular, when $p=1$, we also have $\pi_{1}(M)=0$.

\section{Submanifolds of Euclidean space}

From now on we consider $M^{n}$ to be a compact submanifold of the Euclidean space $\mathbb{R}^{n+m}$.

We shall denote by $\tilde{\nabla}$ the connection on $\mathbb{R}^{n+m}$ and by $T$ and $N$ the tangential and normal projections of vectors in $\mathbb{R}^{n+m}$ to $M$.

The second fundamental form $B \in \operatorname{Hom}(T M \times T M, N M)$ is defined by $B(X, Y)=$ $\left(\tilde{\nabla}_{x} Y\right)^{N}$. It is a symmetric bilinear form.

For any $V \in N M$ the shape operator corresponding to $V$ denoted by $A^{v} \in \operatorname{Hom}(T M, T M)$ is defined by

$$
A^{V}(X)=-\left(\tilde{\nabla}_{X} V\right)^{T}
$$

and we have $A^{v}$ is symmetric and satisfies

$$
\langle B(X, Y), V\rangle=\left\langle A^{v}(X), Y\right\rangle
$$

Now we consider a parallel vector field $V$ in $\mathbb{R}^{n+m} . V^{T}$ is the gradient of the function $f(x)=\langle x, v\rangle$ on $M$ where $x$ denotes the position vector of points on $M$.

We have

$$
\begin{aligned}
\nabla_{e_{i}}\left(V^{T}\right) & =\left(\tilde{\nabla}_{e_{i}}\left(v-v^{N}\right)\right)^{T} \\
& =-\left(\tilde{\nabla}_{e_{i}} V^{N}\right)^{T} \\
& =A^{v^{N}}\left(e_{i}\right) .
\end{aligned}
$$

Therefore

$$
\begin{aligned}
& \left\langle\nabla_{e_{i}}\left(V^{T}\right), e_{i}\right\rangle=\left\langle B\left(e_{i}, e_{i}\right), V^{N}\right\rangle \\
& \left\langle\nabla_{e_{i}}\left(V^{T}\right), n_{k}\right\rangle=\left\langle B\left(e_{i}, n_{k}\right), V^{N}\right\rangle
\end{aligned}
$$


and

$$
\begin{aligned}
\left\|\nabla_{e_{i}}\left(V^{T}\right)\right\|^{2} & =\left\langle A^{v^{N}}\left(e_{i}\right), A^{v^{N}}\left(e_{i}\right)\right\rangle \\
& =\sum_{j}\left\langle A^{v^{N}}\left(e_{i}\right), e_{j}\right\rangle^{2}+\sum_{k}\left\langle A^{v^{N}}\left(e_{i}\right), n_{k}\right\rangle^{2} \\
& =\sum_{j}\left\langle B\left(e_{i}, e_{j}\right), V^{N}\right\rangle^{2}+\sum_{k}\left\langle B\left(e_{i}, n_{k}\right), V^{N}\right\rangle^{2} .
\end{aligned}
$$

Hence substituting in (1), we have

$$
\begin{aligned}
\left\langle\int \sum_{i} B\left(e_{i}, e_{i}\right), V^{N}\right\rangle^{2} & +\sum_{i, k}\left\langle B\left(e_{i}, n_{k}\right), V^{N}\right\rangle^{2}+\sum_{i}\left\langle R\left(V^{T}, e_{i}\right) V^{T}, e_{i}\right\rangle \\
& -\sum_{i, j}\left\langle B\left(e_{i}, e_{j}\right), V^{N}\right\rangle^{2} \geqq 0 .
\end{aligned}
$$

Following [2], we define a bilinear form on $\mathbb{R}^{n+m}$ by

$$
\begin{aligned}
(V, W)= & \int\left\langle\Sigma B\left(e_{i}, e_{i}\right), V^{N}\right\rangle\left\langle\Sigma B\left(e_{i}, e_{i}\right), W^{N}\right\rangle+\sum_{i, k}\left\langle B\left(e_{i}, n_{k}\right), V^{N}\right\rangle\left\langle B\left(e_{i}, n_{k}\right), W^{N}\right\rangle \\
& +\Sigma\left\langle R\left(V^{T}, e_{i}\right) W^{T}, e_{i}\right\rangle-\sum_{i, j}\left\langle B\left(e_{i}, e_{j}\right), V^{N}\right\rangle\left\langle B\left(e_{i}, e_{j}\right), W^{N}\right\rangle
\end{aligned}
$$

and the quadratic form $Q$ on $\mathbb{R}^{n+m}$ by

$$
Q(V)=(V, V)
$$

Then from (2) we have

$$
Q(V) \geqq 0
$$

Hence trace $(Q) \geqq 0$.

To evaluate trace $(Q)$ we observe that it is independent of the choice of orthonormal basis for $\mathbb{R}^{n+m}$ and so at a point $x \in \mathscr{S}$, we choose orthonormal basis $\left\{e_{i}, \ldots, e_{p}, n_{1}, \ldots, n_{q}, f_{1}, \ldots, f_{m}\right\}$ were $e_{i}$ and $n_{k}$ 's are as before and $f_{\alpha}(1 \leqq \alpha \leqq m)$ are normal to $M$. From (2) we have

$$
\begin{aligned}
\operatorname{trace}(Q)= & \int \sum_{i, j}\left\langle B\left(e_{i}, e_{i}\right), B\left(e_{j}, e_{j}\right)\right\rangle+\sum_{i, k}\left\|B\left(e_{i}, n_{k}\right)\right\|^{2}-\sum_{i, j}\left\|B\left(e_{i}, e_{j}\right)\right\|^{2} \\
& +\sum_{i, j}\left\langle R\left(e_{j}, e_{i}\right) e_{j}, e_{i}\right\rangle+\sum_{i, j}\left\langle R\left(n_{k}, e_{i}\right) n_{k}, e_{i}\right\rangle \geqq 0
\end{aligned}
$$


For any two orthonormal vectors $X, Y \in T M_{x}$ we let $K(X, Y)$ denote the sectional curvature corresponding to $X$ and $Y$. So $K(X, Y)=\langle R(X, Y) Y, X\rangle$. We have the Gauss equation

$$
K(X, Y)=\langle B(X, X), B(Y, Y)\rangle-\|B(X, Y)\|^{2} .
$$

So from (3) we have

$$
\int \sum_{i, k}\left(\left\|B\left(e_{i}, n_{k}\right)\right\|^{2}-K\left(e_{i}, n_{k}\right)\right) \geqq 0
$$

or equivalently

$$
\int \sum_{i, k}\left(2\left\|B\left(e_{i}, n_{k}\right)\right\|^{2}-\left\langle B\left(e_{i}, e_{i}\right), B\left(n_{k}, n_{k}\right)\right\rangle\right) \geqq 0 .
$$

From Proposition 1 and (4) we conclude that

Theorem A. Let $M^{n}$ be a compact submanifold of $\mathbb{R}^{n+m}$. If for all $x \in M$ and any two orthonormal vectors $X, Y$ in $T M_{x}$, we have

$$
R(X, Y, X, Y)>\|B(X, Y)\|^{2} .
$$

Then there are no stable p-dimensional currents on $M$ for all $1 \leqq p \leqq n-1$.

\section{Hypersurface with positive sectional curvature in Euclidean space}

We now consider $M^{n}$ to be a compact hypersurface in $\mathbb{R}^{n+1}$. We suppose that the sectional curvatures on $M$ are all positive. Then with respect to a suitably chosen normal direction we may assume the principal curvatures $\lambda_{1}, \ldots, \lambda_{n}$ are all positive and we arrange them so that

$$
0<\lambda_{1} \leqq \lambda_{2} \cdots \leqq \lambda_{n}
$$

We let $e$ be the above chosen unit normal. Let $B\left(e_{i}, e_{j}\right)=h_{i j} e$ and $B\left(e_{i}, n_{k}\right)=h_{i k} e$. Then the $\lambda_{a}$ 's $(a=1, \ldots, n)$ are the eigenvalues of the symmetric $n \times n$ matrix

$$
H=\left(\begin{array}{c|c}
h_{i j} & h_{i k} \\
\hline h_{l j} & h_{l k}
\end{array}\right)
$$

We shall need the following algebraic lemma.

Lemma. Let $H=\left(h_{a b}\right), 1 \leqq a, b \leqq n$ be a symmetric $n \times n$ matrix. Let $\lambda$ be the smallest eigenvalue of $H$. Then $h_{a a} \geqq \lambda$ for all $a=1, \ldots, n$. 
From (5) we have

$$
\int \sum_{i, k}\left(2\left(h_{i k}\right)^{2}-h_{i i} h_{k k}\right) \geqq 0
$$

To estimate the left side of (6) we note that by the lemma we have $h_{i i} \geqq \lambda_{1}>0$ and $h_{k k} \geqq \lambda_{1}>0$. We let $\|H\|^{2}=\Sigma h_{a b}^{2}$, then we have

$$
\begin{aligned}
\int \sum_{i, k} 2\left(h_{i k}\right)^{2} & -\sum_{i, k} h_{i i} h_{k k} \leqq \int\|H\|^{2}-\sum_{i}\left(h_{i i}\right)^{2}-\sum_{k}\left(h_{k k}\right)^{2}-\left(\sum_{i} h_{i i}\right)\left(\sum_{k} h_{k k}\right) \\
& \leqq \int\|H\|^{2}-\frac{1}{n}(\operatorname{trace} H)^{2}-p q \lambda_{1}^{2}=\int \Sigma \lambda_{a}^{2}-\frac{1}{n}\left(\Sigma \lambda_{a}\right)^{2}-p q \lambda_{1}^{2} \\
& \leqq \int \Sigma \lambda_{a}^{2}-\frac{1}{n}\left(\Sigma \lambda_{a}\right)^{2}-(n-1) \lambda_{1}^{2} .
\end{aligned}
$$

Hence, we obtain

Theorem B. For a compact hypersurface $M^{n}$ in $\mathbb{R}^{n+1}$; suppose all the sectional curvature on $M$ are positive and with respect to a suitably chosen normal direction the principal curvatures are arranged so that

$$
0<\lambda_{1} \leqq \lambda_{2} \leqq \cdots \leqq \lambda_{n}
$$

If

$$
\sum_{i=1}^{n} \lambda_{i}^{2} \leqq \frac{1}{n}\left(\sum_{i=1}^{n} \lambda_{i}\right)^{2}+(n-1) \lambda_{1}^{2}
$$

then there are no stable currents on $M$.

Corollary. In the situation of Theorem $B$, if

$$
\frac{\lambda_{1}^{2}}{\lambda_{n}^{2}}>\frac{n}{2 n-1}
$$

then there are no stable currents on $M$.

Now we consider an ellipsoid in $\mathbb{R}^{n+1}$ defined by

$$
a x_{1}^{2}+x_{2}^{2}+\cdots+x_{n+1}^{2}=1
$$

where $a>0$. 
A simple direct computation shows that its principal curvatures are given by

$$
\frac{1}{l}, \ldots, \frac{1}{n-1}, \frac{\lambda}{l}, \frac{\lambda}{l}
$$

where

$$
l^{2}=a^{2} x_{1}^{2}+x_{2}^{2}+\cdots+x_{n+1}^{2}=1+a(a-1) x_{1}^{2}
$$

and

$$
\lambda=\frac{a}{1+a(a-1) x_{1}^{2}} .
$$

To illustrate Theorem B we observe that the condition there is a homogeneous quadratic expression in the $\lambda_{i}$ 's and so we can ignore the common factor $1 / l$.

Since $0 \leqq x_{1}^{2} \leqq 1 / a$, so for $a \geqq 1$ we have $a \geqq \lambda \geqq 1$ and for $a \leqq 1$ we have $a \leqq \lambda \leqq 1$.

Then a direct computation shows the following

Example. There are no stable currents on the ellipsoid

$$
a x_{1}^{2}+x_{2}^{2}+\cdots+x_{n+1}^{2}=1
$$

if

$$
\frac{1}{1+\sqrt{n}}<a<1+\sqrt{n}
$$

Acknowledgement. I wish to thank the referee for many helpful sugggestions.

\section{REFERENCES}

1. R. S. Hamilton, Three-manifolds with positive Ricci curvature, J. Diff. Geom. 17 (1982), 255-306.

2. Lawson and Simons, On stable currents and their application to global problems in real and complex geometry, Ann. of Math. 98 (1973), 427-450.

3. J. D. Moore, Codimension two submanifolds of positive curvature, Proc. A.M.S. 70 (1978), 72-74.

4. E. H. Spanier, Algebraic Topology (McGraw-Hill).

Department of Mathematics

NATIONAL UNIVERSITY OF SiNGAPORE

Kent Ridge

S!NGAPORE 0511 